\title{
Research on Multi-attribute Decision Making Model Based on Possible Regret Degree of Policy-maker
}

\author{
Yan LI \\ Department of Economics, Huaihua College , China \\ liyan77@163.com
}

Keywords: Multi-attribute Decision Making, Plus Regret Degree, Regret Degree Threshold

\begin{abstract}
This article will conduct the research to this question, in order to portray the decision-makers dilemma in the decision-making process under the uncertain condition, this paper defines the following concept: policy-makers plus regret degree, policy-makers negative regret degree and policy-makers weighted combination regret degree. According to the different thresholds of policy-makers plus regret degree, policy-makers negative regret degree and policy-makers weighted combination regret degree, respectively determine the best plan and construct the optimal decision results table, policy-makers according to their preference situation can query this table and select the most excellent plan suited to their own situation.
\end{abstract}

\section{I . Introduction}

In the non-definite multi-attribute decision-making process, because the decision-making information is non-definite, so policy-makers select the best plan needing to face the following dilemma: Suppose there are two alternatives A and B to be available for policy-makers choose, policy-makers choose A and give up B, as decision-making information with non-definite, it actually may be that the B plan is better than A plan. In other words, policy makers in decision-making process with non-definite decision-making information most likely will regret. Existing research about the non-definite multi-attribute decision making problems is rarely related to this question, and this issue is root causes disturbed the policy-maker when caring on policy-making under the non-definite condition. This article will conduct the research to this question, in order to portray the decision-makers dilemma in the decision-making process under the uncertain condition; this paper defines the following concept: policy-makers plus regret degree, policy-makers negative regret degree and policy-makers weighted combination regret degree. According to the different thresholds of policy-makers plus regret degree, policy-makers negative regret degree and policy-makers weighted combination regret degree, respectively determine the best plan and construct the optimal decision results table, policy-makers according to their preference situation can query this table and select the most excellent plan suited to their own situation.

\section{II . Literature References}

In literature [1], defines the following concept: the expectation level of alternative, the uncertain linguistic negative point, the achievement scale, the alternative comprehensive scale under uncertain linguistic environment. Based on these concepts, some linear programming models are established, through which the decision maker interacts with the analyst. In literature [2], A TOPSIS method is proposed to deal with multiple attribute decision-making [MADM] problems with attribute weights unknown completely and the attribute values taken the form of uncertain linguistic variables. In literature [3], with respect to multiple-attribute decision-making (MADM) problems with uncertain linguistic information, a decision analysis method based on linguistic probability is proposed. The conceptofuncertain linguistic variable is introduced and linguistic probabilistic ordered weighted averaging (LPOWA) operator is proposed. In literature [4], study the multiple attribute decision making problems, in which the information about attribute weights is completely unknown and the 
attribute values take the form of uncertain linguistic variables. In literature [5], a simple and explicit formula for obtaining the attribute weights is given by the concept of range extremity difference. In literature [6], a new decision making approach based on D-S theory is proposed for the multi-attribute group decision making problem with incomplete linguistic assessment information. Literature [7]-[10] has conducted the research to the sector multi-objective decision making question: In literature [7], studies a kind of multi-attribute decision-making problem in which the preference information on alternatives and the attribute values are described by interval-valued intuitionist fuzzy numbers, and proposes a new method. In literature [8], for precisely quantify uncertainty, according to the isomorphic fundamental principles and the relative theories and the thought of the similar science, a new algorithm based on an isomorphism multi-objective decision-making new method was proposed. In literature [9], based on the connection number theory of Set Pair Analysis, find the interaction point of certainty and uncertainty, it shows the size of the interaction point. In literature [10], based on the concept of negative ideal point by considering the vector projection, the close-degree of the idealpointand the negative close to the ideal point is comprehensively analyzed.

\section{Basic Concept Synopsis}

This article discusses attribute type, either type of cost attribute (the value of this type attribute is more small, the plan is more good), or the efficiency attribute (the value of this type attribute is more small, the plan is more bad). Moreover, suppose the decision information table to undergo standardized processing in this paper.

Supposes $A=\left\{A_{1}, A_{2}, \cdots, A_{n}\right\}$ as the pre-set, $U=\left\{u_{1}, u_{2}, \cdots, u_{m}\right\}$ as the attribute set. $\widetilde{v}_{k i}$ expresses a sector value $\left[v_{k i}^{L}, v_{k i}^{R}\right]$ obtained by caring on appraising the plan $A_{k}(k=1,2, \cdots, n)$ under the attribute $u_{i}(i=1,2, \cdots, m)$, then may get sector number decision-making matrix $\tilde{V}=\left[\tilde{v}_{k i}\right]_{n \times m}$. Supposes the attribute $u_{1}, u_{2}, \cdots, u_{m}$ expert weight value respectively is $\omega_{1}, \omega_{2}, \cdots, \omega_{m}$, where $\omega_{i} \geq 0$ $(i=1,2, \cdots, m)$, and $\omega_{1}+\omega_{2}+\cdots+\omega_{m}=1$.

Definition 1 . The policy-maker carries on the decision-making under the condition that the attribute value takes the sector value $\tilde{v}_{k i}$, caring on evaluation to plan $A_{k}(k=1,2, \cdots, n)$ under the attribute $u_{i}(i=1,2, \cdots, m)$ obtains the evaluation value $v_{k i j}$. When the attribute $u_{i}$ is an efficient attribute, the policy-maker negative regret degree equals with $\frac{v_{k i}^{R}-v_{k i j}}{v_{k i}^{R}-v_{k i}^{L}}$, records as $h_{k i j}^{-}$. When the attribute $u_{i}$ is a cost attribute, the policy-maker negative regret degree equal with $\frac{v_{k i}^{R}-v_{k i j}}{v_{k i}^{R}-v_{k i}^{L}}$, records as $h_{k i j}^{-}$.

Definition 2. The policy-maker carries on the decision-making under the condition that the attribute value takes the sector value $\widetilde{v}_{k i}$, carring on evaluation to plan $A_{k}(k=1,2, \cdots, n)$ under the attribute $u_{i}(i=1,2, \cdots, m)$ obtains the evaluation value $v_{k i j}$. When the attribute $u_{i}$ is an efficient attribute, the policy-maker plus regret degree equals with $\frac{v_{k i}^{R}-v_{k i j}}{v_{k i}^{R}-v_{k i}^{L}}$, records as $h_{k i j}^{+}$. When the attribute $u_{i}$ is a cost attribute, the policy-maker plus regret degree equals with $\frac{v_{k i j}-v_{k i}^{L}}{v_{k i}^{R}-v_{k i}^{L}}$, records as $h_{k i j}^{+}$. Obviously, $0 \leq h_{k i j}^{-} \leq 1$, as the attribute $u_{i}$ is an efficient attribute, $h_{k i j}^{-}$and $v_{k i j}$ are cocurrently changing; When the attribute $u_{i}$ is a cost attribute, $h_{k i j}^{-}$and $v_{k i j}$ are reversely changing. $0 \leq h_{k i j}^{+} \leq 1$, 
as the attribute $u_{i}$ is an efficient attribute, $h_{k i j}^{+}$and $v_{k i j}$ are reversely changing, When the attribute $u_{i}$ is a cost attribute, $h_{k i j}^{+}$and $v_{k i j}$ are cocurrently changing.

Definition 3. If the policy-makers selects their own negative regret withstanding degree as $h_{k i j}^{-}$, When the attribute $u_{i}$ is an efficient attribute, call $v_{k i}^{L}+h_{k i j}^{-} \times\left(v_{k i}^{R}-v_{k i}^{L}\right)$ as the best value to $A_{k}(k=1,2, \cdots$ $, n)$ under the condition that the policy-maker withstanding negative regret degree value take $h_{k i j}^{-}$. When the attribute $u_{i}$ is a cost attribute, call $v_{k i}^{R}-h_{k i j}^{-} \times\left(v_{k i}^{R}-v_{k i}^{L}\right)$ as the best value to $A_{k}(k=1,2, \cdots$ ,$n$ ) under the condition that the policy-maker withstanding negative regret degree value take $h_{k i j}^{-}$, record as $v_{k i j}^{-}$.

Definition 4. If the policy-makers selects their own plus regret withstanding degree as $h_{k i j}^{+}$, When the attribute $u_{i}$ is an efficient attribute, call $v_{k i}^{R}-h_{k i j}^{-} \times\left(v_{k i}^{R}-v_{k i}^{L}\right)$ as the worst value to $A_{k}(k=1,2, \cdots$ ,$n$ ) under the condition that the policy-maker withstanding plus regret degree value take $h_{k i j}^{+}$. When the attribute $u_{i}$ is a cost attribute, call $v_{k i}^{L}+h_{k i j}^{-} \times\left(v_{k i}^{R}-v_{k i}^{L}\right)$ as the worst value to $A_{k}(k=1,2, \cdots$ ,$n$ ) under the condition that the policy-maker withstanding plus regret degree value take $h_{k i j}^{+}$, record as $v_{k i j}^{+}$.

Defined by the definition 3 and definition 4 we can known, when the policy-makers simultaneously considering the plus regret degree $h_{k i j}^{+}$and the negative regret degree $h_{k i j}^{-}$, to make existing attribute evaluation in the interval evaluation value $\left[v_{k i}^{L}, v_{k i}^{R}\right]$ to satisfy the plus regret degree $h_{k i j}^{+}$and the negative regret degree $h_{k i j}^{-}$, the following inequality $h_{k i j}^{+}+h_{k i j}^{-} \geq 1$ must establish. When $h_{k i j}^{+}+h_{k i j}^{-}=1$, have only one feasible value. When $h_{k i j}^{+}+h_{k i j}^{-}>1$, feasible values constitute a sector.

Definition 5. If the policy-makers selects their own plus regret withstanding degree as $h_{k i j}^{+}$and negative regret withstanding degree as $h_{k i j}^{-}$, When the attribute $u_{i}$ is an efficient attribute, call the sector $\left[v_{k i j}^{+}, v_{k i j}^{-}\right.$]as feasible evaluation value region of the plan $A_{k}(k=1,2, \cdots, n)$ under the attribute $u_{i}(i=1,2, \cdots, m)$. When the attribute $u_{i}$ is a cost attribute, call the sector $\left[v_{k i j}^{-}, v_{k i j}^{+}\right]$as feasible evaluation value region of the plan $A_{k}(k=1,2, \cdots, n)$ under the attribute $u_{i}(i=1,2, \cdots, m)$.

Definition 6. When the policy-makers selects their own plus regret withstanding degree as $h_{k i j}^{+}$and negative regret withstanding degree as $h_{k i j}^{-}$, call $w_{1} \times h_{k i j}^{+}+w_{2} \times h_{k i j}^{-}$as the policy-maker weighted combination regret degree, records as $h_{k i j}$. Where, $0 \leq w_{1}, w_{2} \leq 1$ and $w_{1}+w_{2}=1$.

\section{Constructing Policy-making Algorithm}

Policy-making algorithm

Input: (1) Decision information table. (2) The attribute $u_{1}, u_{2}, \cdots, u_{m}$ expert weight value $\omega_{1}, \omega_{2}, \cdots$ , $\omega_{m}$

(3) The policy-maker acceptable plus (negative) regret degree value.

Output: Policy-making result table

Begin:

The first step: Extracts each set $\widetilde{v}_{1 i} \cup \widetilde{v}_{2 i} \cup \cdots \cup \widetilde{v}_{n i}$ the right endpoint value $v_{i}^{R}$ and left endpoint value $v_{i}^{L},(i=1,2, \cdots, n)$. 
The second step: When the attribute $u_{i}$ is an efficient attribute, extracting the value $v_{k i j}^{+}$to each regret degree value according to the formula $v_{k i}^{R}-h_{k i j}^{-} \times\left(v_{k i}^{R}-v_{k i}^{L}\right)$. When the attribute $u_{i}$ is a cost attribute, extracting the value $v_{k i j}^{+}$to each regret degree value according to the formula $v_{k i}^{L}+h_{k i j}^{-} \times\left(v_{k i}^{R}-v_{k i}^{L}\right)$.

The third step: When the attribute $u_{i}$ is an efficient attribute, extracting the value $v_{k i j}^{-}$to each regret degree value according to the formula $v_{k i}^{L}+h_{k i j}^{-} \times\left(v_{k i}^{R}-v_{k i}^{L}\right)$. When the attribute $u_{i}$ is a cost attribute, extracting the value $v_{k i j}^{-}$to each regret degree value according to the formula $v_{k i}^{R}-h_{k i j}^{-} \times\left(v_{k i}^{R}-v_{k i}^{L}\right)$.

The fourth step: Takes the sector $\left[v_{k i j}^{-}, v_{k i j}^{+}\right]$mid-point value.

The fifth step: Using the average weighting operator to fuse evaluation value to each reelection plan under various attributes, then obtains the synthesis evaluation value.

\section{Summary}

This article has conducted the research to the sector multi-objective decision making question, comparing to existing research to this kind of multi-objective decision making question, the innovation of this paper is: as to policy-maker, under the condition that the decision information is non-definite, possibly may regret after caring on the decision-making, define the following concept: policy-makers plus regret degree, policy-makers negative regret degree and policy-makers weighted combination regret degree. According to the different thresholds of policy-makers plus regret degree, policy-makers negative regret degree and policy-makers weighted combination regret degree, respectively determine the best plan and construct the optimal decision results table, policy-makers according to their preference situation can query this table and select the most excellent plan suited to their own situation.

\section{Acknowledgements}

This paper was financially supported by Hunan Province Philosophy and Social Science Project " Research on the Internet Media Effect China's Stock Market and Network Public Opinion Monitor Index of Listed Companies” (project number: 14YBA306).

This paper was the stage research results of the Hunan province education planning Project "School-enterprise cooperation in personnel training Virtual Alliance: organizational structure, resource sharing and performance assessment study” (project number: XJK015BGD053)

\section{References}

[1] XU Ye-jun, DA Qing-li, ZHAO Chen-xi. “Interactive approach for multiple attribute decision making with incomplete weight information under uncertain linguistic environment”. Systems Engineering and Electronics, 2009(03): 597-601.

[2]LIUPei-de. TOPSIS “Method for Multiple Attribute Decision-making Based on Uncertain Linguistic Variables”. JOURNAL OF WUHAN UNIVERSITY OF TECHNOLOGY, 2009(08): 151-161.

[3]ZHANG Yao, FAN Zhi-ping. "Uncertain LinguisticMultiple Attribute Decision M aking Approach Based on Probability and ItsApplication”. OPERATIONS RESEARCH AND MANAGEMENT SCIENCE, 2009(12): 65-69.

[4]WEI Gui-wu. “Combined Method of Uncertain Linguistic Multiple Attribute Decision Makin”. Fuzzy Systems and Mathematics, 2008(08): 106-111.

[5] WU Z B, ChenYH. "Themaximizing deviationmethod forgroup multiple attribute decision making under linguistic environment, Fuzzy Setsand Systems”. 2007, 158(14): 1608-1617. 
[6]YAO Bing-xue1, ZHANG Fang-wei. “A New Method of Multi-attribute Decision Making Based on Fuzzy Linguistic Assessments”. Fuzzy Systems and Mathematics, 2008(08): 111-123.

[7] GONG Ben-gang, HUA Zhong-sheng, TAN Da-shui. ”A Method of Multi-Attribute Group Decision Making with Incomplete Linguistic Assessment Information”. Chinese Journal of Management Science, 2007(02): 88-92.

[8]SHILi-juan,HHANG Tian-min,CHENG Ya-liA M ethod of Interval-Valued Intuitionistic FuzzyMulti-Attribute DecisionM aking Combined with the Subjective and Objective Preference[J]. Journal ofChongqing Institute ofTechnology(NaturalScience), 2009(11): 158-162.

[9]ZHOU Qi-hai1, LI Yan, HUANG Tao, HAN Zai-xing, LI Yu-jun. New Isomorphic Algorithm of Interval Multi-attributes Decision-making Based on Risk-weighted Similarity[J]. Computer Science, 2009(01): 198-200,221.

[10] LIU Xiu-mei1, ZHAO Ke-qin. Multiple Attribute Decision Making Based on the Interval Numbers Certainty and the Uncertainty Interact on Each Other[J]. MATHEMATICS IN PRACTICE AND THEORY, 2009(04): 68-75.

[11] GAO Shan-lin,YUE Qi, LI Jian, BAIBo. The Method of IntervalMulti-attribute Decision-making Improvement [J]. Journal ofChongqing Institute of Technology (Natural Science), 2009(04): 49-52.

[12] WAN Shu-ping. Method of inclination measurement for interval multi-attribute decision-making [J]. Computer Engineering and Applications, 2009, 45(26): 204-205. 